\title{
Le référendum du 23 juin 2016 en Écosse : entre deux unions
}

The June 2016 Referendum in Scotland: Between Two Unions

\section{Edwige Camp-Pietrain}

\section{(2) OpenEdition}

1 Journals

Édition électronique

URL : http://journals.openedition.org/rfcb/1344

DOI : $10.4000 /$ rfcb. 1344

ISSN : 2429-4373

Éditeur

CRECIB - Centre de recherche et d'études en civilisation britannique

Référence électronique

Edwige Camp-Pietrain, «Le référendum du 23 juin 2016 en Écosse : entre deux unions 》, Revue Française de Civilisation Britannique [En ligne], XXII-2 | 2017, mis en ligne le 30 mai 2017, consulté le 03 mai 2019. URL : http://journals.openedition.org/rfcb/1344; DOI : 10.4000/rfcb.1344

Ce document a été généré automatiquement le 3 mai 2019.

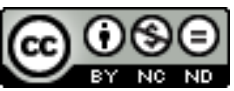

Revue française de civilisation britannique est mis à disposition selon les termes de la licence Creative Commons Attribution - Pas d'Utilisation Commerciale - Pas de Modification 4.0 International. 


\title{
Le référendum du 23 juin 2016 en Écosse : entre deux unions
}

The June 2016 Referendum in Scotland: Between Two Unions

\author{
Edwige Camp-Pietrain
}

\section{Introduction}

1 Dès le Moyen-Age, avant l'union de 1707 avec l'Angleterre, les relations entre l'Écosse et le continent (Scandinavie, Pays-Bas, États allemands, Pologne) étaient multiples, à travers le commerce, l'émigration (soldats, étudiants), voire même les accords politiques (Auld alliance avec la France). Au sein de la Grande-Bretagne, les Écossais se sont recentrés sur les îles britanniques et l'Empire. Au début du XXI ${ }^{e}$ siècle, alors que ce dernier n'est plus qu'un souvenir, et que les premières connaissent des divisions politiques croissantes, les dirigeants écossais mettent en avant le caractère européen de leur nation, dont l'intégration dans l'Union européenne (UE) constitue un symbole ${ }^{1}$.

2 Si cette attitude pro-européenne s'est manifestée à plusieurs reprises, l'appartenance au Royaume-Uni est devenue une source de discorde comme l'a montré le référendum d'autodétermination du 18 septembre 2014 ; près de $45 \%$ de l'électorat ayant opté pour l'indépendance, la population reste profondément divisée sur ce sujet. Pendant la campagne référendaire de 2016, les élus écossais, ainsi que les citoyens, ont continué à affirmer leur différence sur les questions européennes. Mais le vote britannique, en les incitant à repenser les relations avec l'UE et avec le reste du Royaume-Uni, pourrait les conduire à choisir entre les deux.

3 Cet article vise à analyser l'évolution de la perception de la compatibilité entre les deux unions, UE et Royaume-Uni, lors de l'annonce du référendum sur l'UE, pendant la campagne précédant celui-ci et enfin au cours des semaines suivantes, jusqu'à la rentrée parlementaire de septembre 2016. Cela permettra de mettre en évidence l'engagement européen manifeste en Écosse, ainsi que l'instrumentalisation de l'UE pour les besoins de la politique intérieure écossaise dominée par l'enjeu constitutionnel. 


\section{L'Écosse de 2013 à 2015 : une volonté de maintien au sein du Royaume-Uni et de l'UE}

4 Lorsque David Cameron a promis un référendum sur le maintien dans l'UE, les Écossais étaient pro-UE et se sentaient en décalage avec les débats ayant lieu en Angleterre.

\section{Une classe politique devenue europhile}

5 La quasi-unanimité de la classe politique écossaise est récente. Lors du référendum de 1975, de nombreux élus du Parti travailliste, alors dominant en Écosse, ainsi que le SNP, qui venait d'obtenir ses premiers élus à la Chambre des Communes, militaient en faveur d'une sortie de la CEE. À partir de la fin des années quatre-vingt, les branches écossaises des partis britanniques ont suivi l'évolution de leur direction sur les questions européennes, tout en tenant compte des perspectives de création d'entités sub-étatiques à l'intérieur du Royaume-Uni (dévolution). Les Travaillistes écossais préconisaient l'intégration européenne et la dévolution, afin d'échapper aux politiques néolibérales des Conservateurs au pouvoir à Londres. Les Conservateurs écossais, plus obnubilés par leur survie et par la dévolution que par la perte de souveraineté au profit de l'UE, sont restés à l'écart des déchirements du parti en Angleterre. L'attitude pro-européenne et prodévolution des Libéraux-démocrates n'a pas varié. Quant aux dirigeants du SNP, comprenant que la plupart des échanges s'effectueraient au sein du nouveau marché unique, ils se sont mis à prôner l'adhésion à la CEE (puis à l'UE) d'une Écosse indépendante, afin d'éviter son isolement. Ils ont également envisagé d'intégrer la monnaie unique, jusqu'à la crise des dettes souveraines dans la zone euro. Ils demeuraient pragmatiques.

6 La dévolution, mise en place en 1999, a permis aux députés au Parlement écossais de Holyrood de confirmer cet engagement européen. Le Scotland Act de 1998 a en effet transféré aux institutions écossaises des domaines de compétences gérés en partie par l'UE (agriculture, pêche, soutien à l'économie, justice et police), tout en exigeant la compatibilité de chaque loi écossaise avec le droit communautaire. Cependant, c'est le gouvernement britannique qui participe aux conseils européens; le gouvernement écossais est seulement consulté au préalable sur les thèmes le concernant, au sein d'un Joint ministerial committee (JMC) (Europe) ${ }^{2}$. À son arrivée au pouvoir en 2007, le gouvernement SNP a réclamé une meilleure prise en compte des intérêts écossais, notamment sur les questions européennes. À partir de 2010, c'est la politique européenne du pouvoir central qu'il a mise en cause.

\section{L'opposition à la politique européenne du gouvernement Cameron}

L'attitude de David Cameron à l'égard de l'Europe inquiétait les Écossais pour deux raisons.

D'une part, le référendum envisagé par le Premier ministre pouvait entraîner une sortie de l'UE. Or cette probabilité, apparue pendant la campagne précédant le référendum d'autodétermination de 2014, était rejetée par la plupart des membres des deux organisations multipartites, indépendantiste (Yes Scotland, composée du SNP, des verts et 
de multiples entités, appuyée par le gouvernement écossais) et unioniste (Better together, comprenant les partis travailliste, libéral-démocrate et conservateur, avec le soutien du gouvernement britannique).

9 Le gouvernement d'Alex Salmond considérait que seule l'indépendance garantirait le maintien dans l'UE; les Écossais éviteraient ainsi une sortie imposée par le vote des Anglais lors du référendum envisagé par Cameron. Selon Nicola Sturgeon, numéro deux du gouvernement écossais, chargée des Affaires constitutionnelles, la candidature de l'Écosse ne serait qu'une simple formalité puisqu'elle appliquait la totalité de la réglementation européenne. Elle pourrait être traitée selon l'article 48, utilisé pour les modifications de traités ${ }^{3}$. Par contre, d'après les unionistes, en votant pour l'indépendance, les Écossais risquaient de devoir quitter les deux unions. Seul le reste du Royaume-Uni demeurant État-membre, l'Écosse devrait déposer une candidature selon la procédure de droit commun (article 49) ${ }^{4}$. Cela nécessitait des négociations longues, d'autant que le gouvernement écossais entendait conserver les dérogations existantes (euro, accords de Schengen, justice et police) et le rabais sur la contribution au budget communautaire. L'issue était incertaine, ce cas de figure, inédit, suscitant la méfiance des gouvernements aux prises avec des partis sécessionnistes.

Grâce à l'instrumentalisation de l'UE, le SNP minorait les ruptures induites par l'indépendance, tandis que les unionistes les amplifiaient en brandissant la perspective d'une Écosse isolée. Ces derniers se sont déclarés soulagés par un résultat permettant le maintien dans les deux unions.

11 Seuls certains indépendantistes ancrés à gauche (tels Jim Sillars, ex-numéro deux du SNP ${ }^{5}$ , le SSP ou la radical independence campaign) estimaient que l'Écosse devait se libérer des deux unions. Elle pourrait négocier un accord avec l'UE, sur le modèle norvégien de l'EEE (accès au marché unique en échange du versement d'une contribution et de l'acceptation de la libre circulation) ou sur le modèle suisse de l'AELE (au cas par cas). Elle s'affranchirait de la PAC et de la PCP.

D'autre part, David Cameron critiquait la Cour européenne des droits de l'homme (CtEDH) qui veille au respect de la convention éponyme (CEDH). Il souhaitait abroger la loi de 1998 (Human Rights Act, HRA) qui, en incorporant la CEDH dans l'ordre juridique interne, oblige toutes les autorités publiques à tenir compte de la jurisprudence de la CtEDH. Or les députés à Holyrood (à l'exception des Conservateurs) y étaient opposés, pour des raisons juridiques (le HRA étant intégré au Scotland Act de 1998, toutes les lois écossaises doivent être compatibles avec la CEDH) et politiques (ils préféraient un texte conférant des droits identiques à tous les Européens à un bill of rights anglais) ${ }^{6}$. Certes, la CEDH est une émanation du Conseil de l'Europe et non de l'UE, mais elle figure dans le préambule de la Charte des droits fondamentaux de l'UE (reprise dans le traité de Lisbonne). La sortie de l'UE faciliterait l'abrogation du HRA.

Le gouvernement écossais, dirigé par Nicola Sturgeon à partir de novembre 2014, et les députés à Holyrood étaient déterminés à défendre l'UE et la CEDH. À l'issue des élections de mai 2015, ils ont été appuyés par les députés SNP à la Chambre des Communes (parmi lesquels Salmond) qui avaient conquis 56 des 59 sièges écossais, tandis que Cameron avait emporté une majorité absolue ${ }^{7}$. L'opinion publique restait cependant moins enthousiaste que ses élites. 


\section{Une opinion publique plutôt favorable à l'Europe}

14 L'évolution du comportement électoral des Écossais reflète celle des élus. Lors du référendum de 1975, la proportion de votants en faveur de la CEE était plus faible en Écosse $(58,4 \%)$ que sur le plan britannique (67,2\%). Les électeurs des îles Hébrides extérieures et Shetland avaient même voté contre. La CEE paraissait lointaine, les conditions d'adhésion privilégiant les intérêts anglais. Depuis 1979, lors des élections au Parlement européen, les Écossais ont plébiscité les partis europhiles, travailliste (jusqu'en 2004) puis SNP, aux dépens des Conservateurs et du UKIP (qui n'a obtenu son premier siège qu'en 2014).

Les attitudes des électeurs sont toutefois plus mitigées. En 2014-2015, selon les études de NatCen ${ }^{8}$, la proportion de personnes voulant rester dans l'UE, qui excédait $60 \%$ en Écosse, était supérieure de 10 points de pourcentage à celle enregistrée en Angleterre (en excluant les indécis). Mais les Écossais n'étaient pas plus nombreux que les Anglais à réclamer une extension des pouvoirs des institutions européennes. En ce qui concerne les affinités politiques, environ $70 \%$ des électeurs SNP, travaillistes et libéraux-démocrates, mais $49 \%$ des conservateurs, étaient partisans d'un maintien, ce qui ne les distinguait guère des électeurs anglais. Par contre, un fort degré d'identification à sa nation (anglaise ou écossaise) avait plus de chance de conduire au rejet de l'UE en Angleterre qu'en Écosse.

En outre, selon une enquête de Survation pour l'université de Dundee, publiée au printemps 2014, les Écossais n'étaient pas insensibles au programme du UKIP ${ }^{9}: 68,4 \%$ étaient pour le renforcement des contrôles sur l'immigration, $62 \%$ pour la réservation des allocations aux immigrés présents depuis plus de cinq ans, 33,6\% pour un affranchissement de la CtEDH. Ces proportions étaient supérieures à la moyenne parmi les sympathisants SNP (légèrement) et, surtout, parmi les conservateurs.

Cet euroscepticisme, moins marqué qu'en Angleterre, était à peine audible en 2016.

\section{L'Écosse pendant la campagne référendaire de 2016 : l'expression d'une voix nationale pro-UE}

Le gouvernement écossais ne souhaitait pas ce référendum sur l'UE, perçu comme une tentative de régler un problème pour les Conservateurs anglais. Il n'a pas été associé aux renégociations avec l'UE menées par le gouvernement Cameron, en vue de l'accord de février 2016. Il a ensuite dénoncé les modalités d'organisation de ce référendum. À la Chambre des Communes, les députés SNP ont critiqué le corps électoral qui, à la différence du référendum sur l'indépendance de l'Écosse, excluait les jeunes de 16-17 ans et les ressortissants de la plupart des États-membres de l'UE résidant au Royaume-Uni ${ }^{10}$. Ils ont également fustigé l'addition de l'ensemble des voix sur le plan britannique, qui ne permettrait pas de tenir compte des résultats obtenus au sein de chaque nation. De plus, ils ont protesté contre la date de cette convocation, qui, quelques semaines après des élections au Parlement écossais, témoignait d'un mépris des enjeux locaux. Faute de pouvoir s'opposer à ce référendum, les Écossais ont mené une campagne dont la ressemblance avec la campagne britannique n'était que superficielle. 


\section{Des acteurs majoritairement pro-européens}

19 Les structures de campagne étaient calquées sur les structures britanniques ${ }^{11}$. Début février, Scotland Stronger in Europe s'est mise en place, autour de Mona Siddiqui (université d'Édimbourg), Kevin Pringle, chargé de communication (après avoir travaillé pour le SNP), Frank Roy (ancien député travailliste à la Chambre des Communes), directeur et John Edward (ancien chef du bureau du Parlement européen en Écosse), porte-parole. Ils étaient entourés d'un conseil consultatif composé d'experts de la société civile.

20 Mais cette organisation a été éclipsée par les élus écossais. Le 26 mai, la motion proeuropéenne de Sturgeon a reçu le soutien de tous les députés SNP, verts et libérauxdémocrates, de la quasi-totalité des travaillistes et de la grande majorité des conservateurs $^{12}$. Ce consensus s'est manifesté solennellement à l'approche du scrutin par deux appels à voter pour l'UE, lancés par les dirigeants des cinq partis représentés à Holyrood $^{13}$, et par toutes les personnes ayant exercé la fonction de First Minister. Les 59 députés écossais à la Chambre des Communes ont fait campagne pour l'UE, y compris David Mundell, le Secrétaire d'État à l'Écosse. Sur le terrain, les militants étaient toutefois moins actifs que les élites, ce qui peut être lié à l'enjeu européen autant qu'à l'enchaînement des campagnes électorales depuis 2014 ; les dépenses étaient également plus faibles que lors des élections récentes, notamment pour le SNP.

21 Au début de 2016, le camp adverse comptait principalement un porte-parole de Leave.EU pour l'Écosse, Jack Montgomery, et une organisation travailliste, Labour Leave (Nigel Griffiths, ancien député à la Chambre des Communes). Vote Leave s'est structurée en mars, autour d'anciens élus à la Chambre des Communes, tels Tom Harris (porte-parole) et Ian Davidson, travaillistes, Michael Forsyth (conservateur, membre de la Chambre des Lords). Seuls deux députés conservateurs à Holyrood en faisaient partie. En mai, Don Robertson a fédéré les militants SNP hostiles à l'UE, contre la volonté de la direction du parti ${ }^{14}$.

Si la campagne restait centrée sur l'Écosse, Ruth Davidson, leader des Conservateurs, et Sturgeon, toutes deux europhiles et excellentes oratrices, ont pris part aux débats télévisés face à Boris Johnston. Mais le SNP a généralement refusé de se montrer aux côtés des partis britanniques, craignant de subir les conséquences d'une association avec les Conservateurs, comme les Travaillistes à l'issue du référendum de 2014. Le SNP a même accusé ceux-ci de mener une campagne négative (comme en 2014), et d'exagérer les conséquences économiques d'une sortie ${ }^{15}$. Cependant, le gouvernement écossais n'a publié qu'un seul document, succinct (15 pages), consacré aux bénéfices concrets de l'UE, loin de tout idéal européen.

23 Ces élus semblaient exprimer la voix de l'Écosse. Sur 15 sondages organisés à partir de l'annonce de la date du référendum, la proportion de personnes ayant l'intention de voter en faveur de l'UE était comprise entre $59 \%$ et $75 \%$ (en excluant les indécis) ${ }^{16}$. Elle n'a pas régressé en fin de campagne, comme dans l'ensemble du Royaume-Uni, fluctuant davantage en fonction des instituts ( $61 \%$ pour YouGov, $70 \%$ pour TNS). Cette proportion, majoritaire dans toutes les catégories socio-professionnelles, était plus faible parmi les ouvriers selon Panelbase.

24 La presse quotidienne a conforté cet état d'esprit. Certains titres écossais ont appelé à voter en faveur de l'UE : Daily Record (populaire), The Herald et The Scotsman (journaux de qualité), The National (indépendantiste, créé en 2014) ${ }^{17}$. Le Sun, eurosceptique en 
Angleterre, s'est abstenu d'inviter à quitter l'UE dans son édition écossaise, devenue le premier quotidien en Écosse.

Dans ce contexte, les thèmes de campagne ont été abordés sous un angle et un ton distincts en Écosse.

\section{L'enjeu constitutionnel : l'Écosse et l'UE}

Les enjeux constitutionnels, omniprésents, n'étaient pas centrés sur la souveraineté (déjà partagée dans les faits, le Parlement de Holyrood gérant des compétences sans subordination politique à Westminster), ni sur les relations possibles avec l'UE (détaillées pendant la campagne de 2014), mais sur le statut de l'Écosse.

S'agissant de la dévolution, les élus pro-européens étaient satisfaits des relations entre Écosse et UE. Ils souhaitaient toutefois que le gouvernement britannique tienne davantage compte de la position écossaise lors des négociations européennes, comme ils l'avaient souligné au sein de la Commission Smith chargée de formuler des recommandations pour poursuivre la dévolution au lendemain du référendum de $2014^{18}$. En 2016, le gouvernement écossais a de surcroît réclamé une présence plus active de l'Écosse lors de certains conseils ministériels au cours de la présidence britannique de l'UE, prévue pour 2017.

Les partisans d'une sortie estimaient que la dévolution en serait renforcée. D'une part, le Parlement écossais, représentant légitime de la population, s'affranchirait de la Cour de Justice de l'UE (CJUE). Or en décembre 2015, celle-ci avait rendu un avis critiquant (au regard des règles du marché unique) la loi imposant un prix minimum de l'alcool vendu en Écosse. Mais contrairement aux insinuations de Vote Leave, cette loi n'avait pas été invalidée, l'avis informant la procédure judiciaire en cours, auprès des tribunaux écossais. La Scottish whisky association (SWA), à l'origine de la plainte, était pro-UE.

D'autre part, selon ces partisans du retrait de l'UE, la sortie était synonyme de transfert de pouvoirs à Holyrood, lequel recouvrerait automatiquement l'entière maîtrise de l'agriculture, la pêche, la justice et la police, sans perte financière, les subventions programmées par la PAC étant maintenues jusqu'en 2020.

30 En ce qui concerne l'indépendance, Sturgeon avait indiqué dès le printemps 2015 qu'une sortie de l'UE imposée par le vote des Anglais pourrait justifier un second référendum d'autodétermination, si tel était le souhait de l'opinion publique. Pendant la campagne pour les élections au Parlement écossais du 5 mai 2016, les indépendantistes étaient divisés: les Verts, pro-européens, ne considéraient pas ce référendum comme une priorité, à la différence de Solidarity et de RISE ${ }^{19}$ qui étaient eux opposés à l'UE. Les unionistes écartaient tout référendum sur une question réglée en 2014. Mais les Conservateurs écossais étaient plus fermes que les Travaillistes, dont le leader, Kezia Dugdale, avait laissé entendre qu'une sortie de l'UE contre la volonté des Écossais pourrait l'inciter à revoir sa position sur l'indépendance, avant de se rétracter. Les Écossais ont désigné 69 députés indépendantistes pro-européens et 60 unionistes (avec une préférence, inédite, pour les Conservateurs sur les Travaillistes).

31 Pendant la campagne référendaire, Sturgeon, comme Salmond, ont profité de leurs interventions télévisées pour faire la promotion de l'UE tout en rappelant leur objectif indépendantiste. Nombre de dirigeants unionistes les ont alors soupçonnés de pratiquer un double jeu : leurs électeurs n'avaient-ils pas intérêt à voter contre l'UE pour obtenir un 
second référendum? Afin de faire taire ces polémiques, Nicola Sturgeon a exhorté ces derniers à se mobiliser pour l'UE, évoquant même la possibilité, pour l'Écosse, d'emporter l'adhésion sur le plan britannique dans l'hypothèse d'un vote serré en Angleterre. L'accession à l'indépendance dans ces conditions n'était pas celle qu'elle privilégiait. Son ministre Humza Yousaf, directeur de la campagne du parti, a minimisé les chances d'un second référendum.

L'UE était également prisée s'agissant des thèmes socio-économiques.

\section{Les thèmes socio-économiques : l'apport de l'UE}

Tout d'abord, sur le plan économique, le gouvernement écossais a démontré les bienfaits du marché unique ${ }^{20}$. L'Écosse constituait la deuxième région britannique pour les investissements des entreprises étrangères ; $42 \%$ de celles-ci (au nombre de 2310) étaient détenues par des Européens. $42 \%$ des exportations s'effectuaient à destination de l'UE; 300000 emplois leur étaient liés. Vote Leave a rétorqué que le reste du Royaume-Uni était le premier partenaire commercial de l'Écosse, la part du reste du monde étant proche de celle de l'UE.

Les transferts financiers étaient substantiels : 4,6 milliards d'euros grâce à la PAC d'ici 2020, plus de 100 millions pour les industries de la mer. S'agissant des fonds structurels, si l'Écosse avait progressivement perdu l'aide régionale à l'issue de l'élargissement de l'UE en 2004, elle continuait à recevoir des fonds structurels (941 millions en 2014-2020), finançant diverses politiques (formation professionnelle, lutte contre l'exclusion sociale, encouragement aux énergies renouvelables), à l'origine de 40000 emplois en six ans. Mais selon les europhobes, l'Écosse, contributeur net au budget communautaire, n'en avait plus besoin ${ }^{21}$.

35 Les groupes de pression étaient majoritairement pro-européens même s'ils dénonçaient une réglementation tatillonne ${ }^{22} .70 \%$ des chefs d'entreprises interrogés par les Chambres de commerce étaient favorables à l'UE, ainsi que COSLA, organisation représentant la plupart des collectivités territoriales. La SWA a salué la protection des noms géographiques, restant insensible à l'accent mis par Vote Leave sur les droits de douane avec les pays tiers. NFU, syndicat agricole, a souligné l'importance des subventions pour ses adhérents ${ }^{23}$. Les dirigeants politiques ont repris ces arguments, pour les opposer aux incertitudes d'un Brexit, comme l'a rappelé Davidson lors du dernier débat de la $\mathrm{BBC}^{24}$.

Les pêcheurs se sont démarqués de ce consensus. Ils affirmaient que les restrictions imposées depuis l'adhésion à la CEE (afin de partager leurs eaux riches en ressources halieutiques avec les pêcheurs d'autres États-membres et de préserver le renouvellement des espèces) leur avaient fait perdre $50 \%$ de leurs effectifs et deux-tiers de leurs bateaux de plus de 10 mètres. La récente régionalisation de la PCP tout comme les augmentations ponctuelles de quotas leur paraissant insuffisantes, ils voulaient retrouver la maîtrise de leurs eaux territoriales afin de négocier des accords avec les pays voisins (Norvège, Islande, îles Féroé). Le 15 juin, ils l'ont fait savoir en participant à la flottille qui a remonté la Tamise, à l'initiative de Nigel Farage.

Ensuite, sur le plan social, le gouvernement écossais a mis en avant la Charte de l'UE sur les droits fondamentaux (traitement équitable des salariés) et la réglementation de l'UE (durée du travail, droit aux congés) ${ }^{25}$. Le mouvement syndical (UNISON, UNITE, GMB, confédération STUC) a loué l'existence d'un socle minimal de protection, assorti de 
marges d'interprétations nationales. Certes, le Royaume-Uni disposait de sa législation avant son entrée dans la CEE, mais les textes européens avaient amélioré la sécurité au travail, la codification, tout en réduisant les différences entre salariés (temps partiel, détachés). Le durcissement récent de la législation était imputable au gouvernement Cameron.

Même les effets sociaux de la réglementation européenne en matière de concurrence n'étaient pas unanimement décriés. Certes, le gouvernement écossais avait dû soumettre à appels d'offres les lignes maritimes entre l'Écosse et ses îles (au grand dam de RMT) ${ }^{26}$. Mais dans un autre contexte, il avait pu sauver les sites sidérurgiques de Clydebridge et Dalzell.

Enfin, le thème de l'immigration a suscité des divergences de fond des deux côtés de la rivière Tweed. Les personnes nées à l'étranger représentaient $7 \%$ de la population écossaise. Parmi elles se trouvaient 173000 citoyens de l'UE (les Polonais étant les plus nombreux). Or depuis les débuts de la dévolution, les dirigeants écossais, et la majorité des élus (Conservateurs inclus), estimaient que la liberté de circulation était avantageuse pour l'économie ( $5 \%$ de la main-d'œuvre, $30 \%$ dans l'agro-alimentaire) et pour la société. Ils entendaient encourager l'immigration, pour des raisons démographiques et éthiques ${ }^{27}$.

Les dirigeants écossais fustigeaient les discriminations, qu'il s'agisse des restrictions aux versements des allocations obtenues par Cameron, ou de la perspective, mise en avant par les partisans du Brexit, de réintroduction de droits universitaires pour les ressortissants de l'UE. Ils pouvaient compter sur le soutien de présidents d'universités qui, peu désireux d'utiliser ce moyen pour accroître leurs ressources, ont souligné l'apport des étudiants de l'UE à l'économie (156 millions) et l'importance des fonds européens pour la recherche.

41 L'étranger ne faisait pas figure de bouc-émissaire. Ce sont les politiques d'austérité du gouvernement Cameron qui étaient responsables des difficultés de nombreux citoyens, comme l'a souligné Sturgeon lors du débat sur ITV, notamment à propos du système de santé (NHS) ${ }^{28}$. Le UKIP était peu audible, son porte-parole, le député au Parlement européen David Coburn, étant frappé d'ostracisme par les autres élus.

Les résultats du référendum ont incité ces élus à revoir leurs priorités.

\section{L'Écosse à l'issue du référendum face à un dilemme entre les deux unions}

43 Le résultat du référendum, distinct du reste du Royaume-Uni, a ré-ouvert le dilemme entre les deux unions.

\section{Des résultats distinguant l'Écosse}

Le 23 juin 2016, 62 \% des Écossais ont opté pour le maintien dans l'UE. Cette majorité s'est retrouvée dans chacune des 32 collectivités territoriales. Cependant, l'Écosse n'a pas totalement échappé à l'euroscepticisme.

Tout d'abord, le taux de participation $(67,2 \%)$ était inférieur à la moyenne britannique (72,2\%) et nettement en-deçà du taux enregistré lors du référendum de 2014 (84,6 \%). Cela peut s'expliquer autant par un manque d'intérêt pour l'enjeu que par une lassitude à l'égard des urnes. 

souhaitaient la quitter. Les majorités en faveur du maintien dans l'UE étaient inférieures à la moyenne écossaise dans un peu plus de la moitié des collectivités territoriales. Dans l'une d'entre elles (Moray), dans laquelle la pêche constitue une activité importante (Peterhead accueillant $50 \%$ des prises écossaises en tonnage), le vote pro-UE l'a emporté de justesse $(50,1 \%)$. Quant à l'allégeance partisane, si l'UE a été plébiscitée par les électeurs libéraux-démocrates, travaillistes et SNP, ces derniers étaient proportionnellement moins enthousiastes ${ }^{29}$.

47 Enfin, la sortie de l'UE était davantage prisée dans les territoires et quartiers défavorisés. Sur l'ensemble des collectivités territoriales, les corrélations sont négatives entre le vote pour le maintien et les indicateurs de difficultés socio-économiques comme la proportion de personnes sans diplôme $(-0,45)$ ou en maladie de longue durée $(-0,29)^{30}$. La corrélation est également négative $(-0,69)$ avec le vote UKIP aux élections européennes de mai 2014 (dépouillées dans les mêmes collectivités territoriales). Le 23 juin, de nombreux Écossais ont donc utilisé leur vote contre l'UE pour protester contre une élite lointaine. une sortie de l'UE contre la volonté des Écossais.

\section{Comment se maintenir dans le Royaume-Uni et dans l'UE?}

Nicola Sturgeon a multiplié les démarches depuis le scrutin. Jugeant la situation inacceptable d'un point de vue démocratique, elle entend explorer toutes les options afin de trouver une solution permettant aux Écossais de conserver une « place » dans l'UE.

Tout d'abord, le gouvernement écossais a pris contact avec l'UE $\mathrm{I}^{31}$. Sturgeon s'est invitée à Bruxelles pendant un sommet européen pour une image symbolique: Cameron a pris congé de ses homologues le 28 et elle a fait son apparition le 29. Au Parlement européen, où Alyn Smith, député SNP, avait été ovationné à l'issue d'un discours pressant l'UE de «ne pas laisser tomber l'Écosse $»^{32}$, elle a pu s'entretenir avec le président, Martin Schultz. Sturgeon a été reçue par le président de la Commission européenne, Jean-Claude Juncker, alors qu'en 2014, le prédécesseur de ce dernier était moins avenant. Cependant, les chefs d'État et de gouvernement ont rappelé qu'ils ne pouvaient négocier qu'avec l'État-membre en le laissant régler ses problèmes internes. De ce fait, Donald Tusk, président du conseil, a refusé de discuter avec Sturgeon. Fergus Ewing a pris part à une réunion des ministres de l'Agriculture.

51 Qui plus est, Sturgeon a ensuite rencontré le président de l'Irlande, des ministres allemand et français. Ses ministres Fiona Hyslop (Affaires extérieures) et Alasdair Allan (Développement international), ont dialogué avec des ambassadeurs et des consuls d'États-membres, afin de rassurer les ressortissants de l'UE vivant en Écosse, en particulier les étudiants (absence de droits universitaires) et les investisseurs. Leur attitude contrastait avec l'ambiguïté des dirigeants britanniques.

52 Cette action para-diplomatique pourrait porter ses fruits sur le moyen terme ${ }^{33}$. Elle a été bénéfique pour l'image de l'Écosse auprès des citoyens européens, car selon un sondage YouGov, $71 \%$ des Allemands et $61 \%$ des Français souhaitaient lui réserver une place dans $\mathrm{l}^{\prime} \mathrm{UE}^{34}$.

Ensuite, Sturgeon a voulu garantir la participation de l'Écosse aux négociations relatives à la sortie de l'UE et aux nouvelles relations avec celle-ci. Dès l'annonce des résultats,

Revue Française de Civilisation Britannique, XXII-2 | 2017 
Cameron avait promis d'associer les nations. Theresa May s'est engagée à rechercher un accord au sein du Royaume-Uni avant l'activation de l'article 50. Cette recherche ne valant cependant pas obtention, l'Écosse ne disposera pas d'un veto. Or tout accord propre à l'Écosse a été écarté par David Davis, ministre en charge de la sortie.

Sturgeon s'est rapprochée des représentants pro-européens de deux territoires ayant voté pour le maintien dans l'UE (le maire de Londres et le chef du gouvernement de Gibraltar), puis de l'ensemble du British-Irish Council. Elle réclame, aux côtés de ses homologues gallois et nord-irlandais, une prise en compte de leurs intérêts dans les décisions en vue des négociations, allant au-delà de simples consultations.

De plus, le Parlement écossais entend être consulté lors du déclenchement de l'article 50 puis sur les accords négociés avec l'UE, par l'intermédiaire d'une legislative consent motion (LCM). Cette coutume s'est imposée lorsque le Parlement britannique s'apprête à légiférer dans un domaine dévolu ou à modifier les compétences du Parlement ou du gouvernement écossais; elle figure désormais dans le Scotland Act de 2016 (issu des travaux de la Commission Smith), mais uniquement pour le premier motif ${ }^{35}$. Or non seulement la sortie affecte nombre de compétences dévolues, mais elle contrevient également à la loi fondatrice des institutions écossaises. Cependant, en l'absence de constitution codifiée, les avis divergent, certains juristes soutenant que le Parlement britannique n'aura pas à se prononcer sur une question de politique étrangère relevant de la prérogative royale. Quant aux effets d'un refus de LCM, les nationalistes l'assimilent à un veto, de nature politique. Mais juridiquement, la Chambre des Communes, souveraine, pourrait passer outre.

Enfin, les dirigeants écossais vont tenter d'affiner leurs exigences. Sturgeon a nommé un conseil présidé par un économiste (Anton Muscatelli) et composé de fins connaisseurs de l'UE, tels David Edward, ancien juge à la CJUE, John Kerr, ancien diplomate, Anne Glover (qui a conseillé le président de la Commission européenne), Alyn Smith et David Martin (respectivement députés SNP et travailliste au Parlement européen), David Frost (SWA). Ils devront évaluer, par des avis ponctuels, l'impact pour l'Écosse de la sortie du Royaume-Uni de l'UE et tenter d'imaginer des solutions pour défendre les intérêts écossais.

57 Le gouvernement écossais, David Mundell, ainsi que les commissions parlementaires (Affaires européennes à Holyrood et Affaires écossaises à la Chambre des Communes) ont entamé des consultations avec des groupes de pression sur la défensive, cherchant à protéger leurs acquis (financement, accès au marché unique) tout en réclamant une révision de la réglementation. La tâche s'annonce ardue avec le secteur de la pêche, les pêcheurs espérant un nouveau départ tandis que les exportateurs sont réservés ${ }^{36}$.

Ces réflexions sont brouillées par le manque de clarté du gouvernement britannique. Un accord « modéré » (modèle norvégien), privilégié en Écosse, semble peu réaliste. Face à la perspective d'un accord plus «dur» (hard Brexit), certains estiment que le Parlement écossais pourrait rester fidèle à la réglementation de l'UE dans ses domaines de compétence, voire conserver l'obligation de compatibilité de ses lois avec les textes européens ${ }^{37}$. Quelques élus ont relancé l'idée de transformation du Royaume-Uni en État fédéral, permettant à chaque composante d'entretenir ses relations avec l'UE, comme le font certains États fédérés allemands, ce qui rejoint des réflexions menées par des parlementaires britanniques, tel Lord Falconer ${ }^{38}$. 
Cependant, le 25 juillet, Sturgeon a prononcé un discours peu conciliable avec un accord « dur » car elle entendait protéger cinq intérêts : le résultat, le marché unique, la libre circulation et les droits sociaux, le règlement commun des grands problèmes du siècle, l'influence sur la réglementation européenne ${ }^{39}$. Elle a mis au défi le gouvernement britannique de profiter de cette rupture pour inventer des solutions et démontrer l'importance de l'Écosse. À défaut, celle-ci pourrait à nouveau être tentée par l'indépendance.

\section{Quelle perspective d'indépendance pour l'Écosse?}

60 Selon Sturgeon, le vote de septembre 2014, en faveur d'une Écosse dans le Royaume-Uni et dans l'UE, ayant été trahi, le Parlement écossais doit pouvoir proposer un référendum sur l'indépendance d'ici 2019 (dans le délai prévu par l'article 50 pour les négociations de sortie) s'il estime que c'est la meilleure façon de protéger la place de l'Écosse dans l'UE. Elle a ordonné à ses fonctionnaires de préparer un avant-projet de loi. Elle va devoir affronter de multiples obstacles, dans une configuration mouvante.

61 Sur le plan politique, l'une des conditions posées par Sturgeon n'est pas remplie: l'opinion publique ne réclame pas ce référendum ${ }^{40}$. Certes, deux sondages organisés après le référendum ont fait apparaître des majorités en faveur de l'indépendance ( $52 \%$ selon Panelbase et Survation). Quelques semaines plus tard, une fois l'émotion dissipée, cette proportion a retrouvé le niveau antérieur au 23 juin ( $47 \%$ selon YouGov).

62 La légitimité de ce référendum ne fait pas l'unanimité au sein du SNP. Selon Gordon Wilson et Jim Sillars, le gouvernement écossais est dépourvu de mandat car non seulement son engagement lors des élections de mai 2016 restait vague mais qui plus est, il a perdu sa majorité absolue ${ }^{41}$. Inversement, Salmond a évoqué un mandat moral à $l^{\prime}$ issue du référendum ${ }^{42}$. Ses collègues se sont référés à la souveraineté du peuple écossais pour justifier cette consultation en dernier recours (et réitérer leur rejet de tout bill of rights $)^{43}$. C'est l'un eux, Angus Robertson, nouveau numéro deux du SNP, qui sera chargé de la stratégie. Il devra notamment calmer les ardeurs des adhérents, qui ont afflué au lendemain du 23 juin (comme en 2014), portant les effectifs à plus de 120000.

Les unionistes se posent en défenseurs des deux unions. Le 28 juin, la motion de Sturgeon demandant le soutien du Parlement écossais dans son exploration de solutions a été endossée par les Travaillistes et les Libéraux-démocrates. Les Conservateurs se sont abstenus parce qu'elle n'excluait pas la convocation d'un référendum d'autodétermination. Ce dernier divise les Travaillistes écossais, leur numéro deux, Alex Rowley, semblant prêt à le cautionner. Tous les unionistes invitent le gouvernement écossais à utiliser les pouvoirs issus du Scotland Act de 2016 pour contrer les effets du Brexit sur le plan économique.

Sur le plan juridique, ce référendum nécessiterait l'autorisation du Premier ministre May, peu probable en l'état. De plus, l'indépendance de l'Écosse reposerait deux questions relatives à l'adhésion à l'UE. S'agissant des modalités, il existe un précédent, en sens inverse. En entrant dans la CEE, le Danemark a autorisé le Groenland à organiser un référendum sur le sujet ${ }^{44}$. Ce dernier optant pour une sortie, effective en 1985, la Commission européenne s'est adaptée (pêche). Certes, l'Écosse ne pourrait prétendre au statut d'État continuateur du Royaume-Uni ; mais elle aurait intérêt à négocier un accord 
concomitant à la sortie de celui-ci, relevant de l'article 50 (décisions à la majorité) plutôt qu'un accord ultérieur (l'article 49 exige l'unanimité).

S'agissant des conditions, outre les questions de justice et de police, immédiatement problématiques (notamment le mandat d'arrêt européen), deux aspects, controversés en 2014, se présenteraient en termes nouveaux. La frontière avec l'Angleterre deviendrait réelle, mais des accords privilégiés pourraient être envisagés (sur le modèle de la Suède et la Norvège). Quant à la monnaie, conserver celle d'un État extérieur à l'UE serait délicat, voire dommageable. Sturgeon ayant exclu l'euro, trois députés à la Chambre des Communes ont lancé une réflexion sur une devise écossaise.

Sur le plan économique, l'indépendance de l'Écosse paraît plus utopique en 2016, à cause de l'effondrement des cours du pétrole ${ }^{45}$. Cependant, Nicholas MacPherson, ex-secrétaire du Trésor, qui avait émis des doutes en 2014, a laissé entrevoir des possibilités de croissance, à condition de lever les incohérences du programme économique ${ }^{46}$. Certains dirigeants d'entreprises pourraient être tentés de délocaliser leur siège social en Écosse, par exemple dans le secteur financier, afin de conserver le passeport européen. L'Écosse pourrait ainsi limiter son déficit rapporté à son PIB (plus de $8 \%$ ).

\section{Conclusion}

Le référendum sur l'UE, destiné à régler une question anglaise, interne au Parti conservateur, a permis au SNP d'obtenir en 2016 ce qu'il souhaitait en 1975, un résultat distinguant l'Écosse de l'Angleterre sur le plan international. De plus, même si le vote britannique ne correspond pas aux attentes de ses dirigeants, il sert leur objectif indépendantiste. Les valeurs de tolérance et d'ouverture étant bafouées, ce sont les fondements du Royaume-Uni qui sont ébranlés. Dans cette optique, les Écossais doivent quitter une union repliée sur elle-même dans laquelle ils ne se reconnaissent plus, afin de rester dans l'UE.

Cependant, si Sturgeon peut ainsi réaffirmer l'intérêt de l'indépendance, elle a conscience des obstacles juridiques et politiques restant à surmonter. Elle a reconnu que l'un des déterminants du vote a été le rejet de l'UE en tant que bouc-émissaire de difficultés socioéconomiques communes aux Britanniques, qui trouvent une expression différente en fonction du contexte social. Elle devra en tenir compte dans la révision de son projet indépendantiste qui ne peut uniquement viser les investisseurs. Environ 400000 personnes ont voté pour l'indépendance de l'Écosse et contre l'UE, parmi lesquelles une forte proportion d'ouvriers. Sturgeon devra aussi tenir composer avec les diverses sensibilités au sein de son parti, car à l'issue du scrutin, quelques élus nationaux ont admis avoir choisi le Brexit le 23 juin.

Qui plus est, si le gouvernement écossais a semblé agir de manière ordonnée dans les jours suivant le référendum, c'est surtout par contraste avec les démissions enregistrées au sommet de l'État britannique. Les élus SNP ont occupé le terrain, à tous les niveaux, portant la voix de l'Écosse tout en s'adaptant au contexte évolutif, aux alliés de circonstance. Mais ce n'est que fin août que Sturgeon a désigné un ministre en charge des négociations avec le Royaume-Uni relatives à la place de l'Écosse dans l'UE (Michael Russell). La définition même de cette dernière a évolué, l'adhésion cédant la place à un simple statut. Le consensus à Holyrood, qui légitime l'action du gouvernement écossais, 
n'est plus aussi solide qu'à la fin de mois de juin, notamment parce que le SNP ne cesse d'évoquer l'accession à l'indépendance en dernier recours.

Même si Sturgeon affiche sa détermination, son calendrier et ses propositions seront influencés par les orientations retenues à Londres (sur lesquelles l'opacité demeure en dépit des questions pressantes des élus SNP qui cherchent à fédérer les députés pro-UE ${ }^{47}$ ). Cela concerne notamment la possibilité d'une reconnaissance des spécificités de l'Écosse dans l'accord avec l'UE ${ }^{48}$, voire d'un transfert de compétences au Parlement écossais dans les domaines controversés (accords commerciaux internationaux, immigration) ${ }^{49}$. Quant à la date d'un second référendum d'autodétermination, attendre l'issue des négociations de sortie permettrait de clarifier les enjeux, mais dans un contexte politique peut-être moins porteur. Une date anticipée (2017) permettrait à Sturgeon de choisir un moment opportun, et éventuellement de se rapprocher de l'UE, mais sur des bases incertaines ${ }^{50}$. $\mathrm{Au}$ final, les électeurs risquent d'être invités à procéder à un arbitrage entre deux unions, selon l'état de chacune d'entre elles au moment de la consultation.

Edwige Camp-Pietrain est Professeur des Universités à l'université de Valenciennes et chercheur au laboratoire CALHISTE (EA 4343). Ses recherches portent sur la vie politique, les institutions et les politiques publiques dans l'Écosse contemporaine. Elle est l'auteur de plusieurs ouvrages parmi lesquels L'Impossible indépendance écossaise? (Atlande, octobre 2014).

\section{BIBLIOGRAPHIE}

AVERY Graham, « Scotland and the European Union », Bruxelles, European Policy Centre, 14 juillet 2016.

DONNELLY Brian, « European Citizens Give Backing to Place in Europe for Scotland », The Herald, 12 juillet 2016, p. 1.

GALlagher Jim, The Brexit Shambles : Charting a Path through the Rubble, Londres, Constitution unit, 21 juillet 2016 .

HM GOVERNMENT (HMG), Scotland Analysis : EU and International Issues, Londres, Stationery Office, Cm 8765, janvier 2014.

HMG, HM Treasury Analysis : the Immediate Economic Impact of Leaving the EU, Londres, Stationery

Office, Cm 9292, mai 2016.

HOUSE OF COMMONS (HC), Hansard, 9 juin 2015.

HC, Hansard, 24 mai 2016.

HC, Hansard, 27 juin 2016.

HC, Westminster Hall, Hansard, 21 juillet 2016.

HC, Hansard, 6 septembre 2016.

HC, SCOTTISH AFFAIRS COMMITTEE (SAC), oral evidence, 9 mai 2016. 
HC, SAC, oral evidence, 25 mai 2016.

HOUSE OF LORDS, EUROPEAN UNION COMMITTEE, The Process of Withdrawing from the European Union, HL paper 138, Londres, Stationery Office, mai 2016.

KEATING Michael, written evidence, HC, SAC, avril 2016.

MAHONAY Daniel et KNox Tim, « Scotland : Could it Become Greece without the Sun? ", Economic Bulletin, Londres, Centre for policy studies, $n^{\circ}$ 80, 2 juillet 2016.

Monteith Brian, « The Case for Brexit » in Jeffery Charlie et Perman Ray (dir.), Britain's Decision. Facts and Impartial Analysis for the EU Referendum on 23 June 2016, Édimbourg, David Hume Institute, 2016, p. 48-52.

MURKENS Jo Eric, JONES Peter et KEATING Michael, Scottish Independence: a Practical Guide, Édimbourg, Edinburgh University Press, 2002.

ORMSTON Rachel, Disunited Kingdom? Attitudes to the EU across the UK, Londres, NatCen Social Research, 2015.

SCOTLAND InSTITUTE, EU Membership: Workplace Rights, Consumer Protection and Legal Safeguards, Glasgow, Scotland Institute, juin 2016.

SCOTTISH GOVERNMENT (SG), Scotland in the European Union, Édimbourg, novembre 2013.

SG, Scotland's Future and Scottish Fisheries, Édimbourg, août 2014.

SG, The Benefits of Scotland's EU Membership, Édimbourg, mai 2016.

SCOTTISH PARLIAMENT (SP), Official Report, 11 novembre 2014.

SP, Official Report, 26 mai 2016.

SP, Official Report, 31 mai 2016.

SP, Official Report, 16 juin 2016.

SP, Official Report, 28 juin 2016.

SP, Official Report, 30 juin 2016.

SP, Official Report, 7 septembre 2016.

SP, EUROPEAN AND EXTERNAL RELATIONS COMMITTEE (EERC), EU Reform and the EU Referendum:

Implications for Scotland, Édimbourg, SP Paper 978, mars 2016.

SP, EERC, Official Report, 30 juin 2016.

SP, EERC, Official Report, 28 juillet 2016.

SP, EERC, Report, 12 septembre 2016.

SP, EERC, Official Report, 14 septembre 2016.

SILLARS Jim, In Place of Failure, Glasgow, Vagabond Voices, 2015.

STURGEON Nicola, DUGDALE Kezia, DAVIDSON Ruth, RENNIE Willie et HARVIE Patrick, « Why We Say Stay », Daily Record, 22 juin 2016.

STURGEON Nicola « Scotland in EU », Édimbourg, Scottish Government, 25 juillet 2016.

WILSON Gordon, « Scotland's Role in the EU », Options for Scotland, février 2016.

WILSON Gordon, « Long Term Strategy for Independence », Options for Scotland, juillet 2016. 
www.whatscotlandthinks.org, consulté le 5 septembre 2016.

\section{NOTES}

1. Si le titre de cet article rappelle celui d'un ouvrage paru en 2005 (DARDANELL Paolo, Between two Unions. Europeanisation and Scottish Devolution. Manchester, Manchester University Press, 2005), le contexte a radicalement changé depuis lors.

2. Les réunions de ce JMC, rassemblant les ministres écossais, gallois, nord-irlandais, sous la présidence du ministre britannique des Affaires extérieures ou de l'Europe, précèdent les conseils européens; les propos échangés demeurent confidentiels. De plus, à la demande du gouvernement britannique, les ministres du gouvernement écossais peuvent représenter le Royaume-Uni lors de conseils des ministres européens portant sur leurs domaines de compétence ; ils sont alors tenus de défendre la ligne britannique.

3. SG, Scotland in the European Union, Édimbourg, novembre 2013, 3.7. Il s'autorisait malgré tout quelques critiques de l'UE.

4. HMG, Scotland Analysis: EU and International Issues, Londres, Stationery Office, janvier 2014, chap. 3.

5. Après avoir contribué à la conversion du SNP à la CEE, il désapprouvait le changement de nature de celle-ci. SILLARs Jim, In Place of Failure, Glasgow, Vagabond Voices, 2015, p. 15.

6. SP, Official Report, 11 novembre 2014, col. 27-31.

7. HC, Hansard, 24 mai 2016, col. 479, Joanna Cherry.

8. ORMSTON Rachel, Disunited Kingdom? Attitudes to the EU across the UK, Londres, NatCen Social Research, 2015, annexes.

9. GARDHAM Magnus, « 7 out of 10 Scots Back UKIP Policy on Immigration' », The Herald, 21 mai 2014, p. 1.

10. HC, Hansard, 9 juin 2015, col. 1071, Alex Salmond.

11. HC, SAC, 25 mai 2016, auditions de Edward et Harris.

12. SP, Official Report, 26 mai 2016, col.9-51. 9 conservateurs et 2 travaillistes ont choisi le rejet ou l'abstention.

13. STURGEON Nicola, DUGDALE Kezia, DAVIDSON Ruth, RENNIE Willie et HARVIE Patrick, « Why We Say Stay », Daily Record, 22 juin 2016.

14. Herald, "SNP Activists Defy Party Line and Launch Campaign for a Vote to Leave », 2 juin 2016, p.6.

15. Ils prévoyaient une hausse de 50000 chômeurs en Écosse sur deux ans, HMG, HM Treasury Analysis: the Immediate Economic Impact of Leaving the EU, Londres, Stationery Office, mai 2016, p. 50.

16. www.whatscotlandthinks.org, consulté le 5 septembre 2016.

17. Les éditoriaux respectifs du 23 juin étaient explicites: « Go and vote Remain », " More to be gained by [...] remaining in the $E U$ ", "The brighter future lies with remaining part of Europe ", "Deliver an emphatic Remain vote ».

18. Sмith Commission, Report of the Smith Commission for Further Devolution of Powers to the Scottish Parliament, Édimbourg, novembre 2014, p. 15.

19. Solidarity est issu du Scottish Socialist Party, lequel avait émergé de la tendance Militant au sein du Parti travailliste dans les années quatre-vingt-dix. RISE a vu le jour après le référendum de 2014, afin de rassembler des indépendantistes ne se reconnaissant pas dans le SNP.

20. SG, The Benefits of Scotland's EU Membership, Édimbourg, mai 2016, chapitre 1.

21. Argument d'un ancien député conservateur à Holyrood, chargé de la communication de Leave.EU, MONTEITH Brian, «The Case for Brexit » in Jeffery Charlie et Perman Ray (dir.), Britain's 
Decision. Facts and Impartial Analysis for the EU Referendum on 23 June 2016, Édimbourg, David Hume Institute, 2016, p. 49.

22. HC, SAC, 9 mai 2016.

23. Ceux-ci venaient de rencontrer des difficultés, en raison des retards de versement. sP, official Report, 31 mai 2016, col. 3.

24. EU Great Debate, Wembley, BBC, 21 juin 2016.

25. SG, The Benefits of Scotland's EU Membership, Édimbourg, mai 2016, chapitre 2.

26. sP, Official Report, 26 mai 2016, col. 50, Elaine Smith (travailliste).

27. sP, Official Report, 16 juin 2016, col. 27-36.

28. EU Debate, ITV, 9 juin 2016.

29. Enquêtes de Lord Ashcroft (www.lordashcroftpolls.com, consulté le 3 septembre 2016) et de Survation (The Scotsman, 5 juillet 2016).

30. Calculs de l'auteur.

31. sP, Official Report, 30 juin 2016, col. 2, Fiona Hyslop.

32. «But, please, remember this : Scotland did not let you down. Please, I beg you, chers collègues, do not let Scotland down now », 28 juin (www.alynsmith.eu, consulté le 3 juillet 2016).

33. Avis d'un directeur-général honoraire de la Commission européenne, AVERY Graham, «Scotland and the European Union », Bruxelles, European Policy Centre, 14 juillet 2016.

34. DONNELly Brian, «European Citizens Give Backing to Place in Europe for Scotland », The Herald , 12 juillet 2016, p. 1.

35. L'intérêt d'une LCM est reconnu au-delà du SNP, par exemple par Sir David Edward, HOUSE OF LORDS, EUROPEAN UNION COMMITTEE, The Process of Withdrawing from the European Union, Londres, Stationery Office, mai 2016, paragraphe 70.

36. SP, EERC, 28 juillet 2016. Les pêcheurs (SFF) s'inquiètent néanmoins du faible poids de leur secteur à l'échelle britannique.

37. Suggestion d'Adam Tomkins, juriste et député conservateur, SP, Official Report, 28 juin 2016, col. 26.

38. Cité par Ian Murray, député travailliste écossais, HC, Hansard, 21 juillet 2016. Selon un ancien leader du SNP, l'Écosse pourrait ainsi adhérer à l'EEE, WILSON Gordon, «Scotland's Role in the EU », Options for Scotland, février 2016.

39. STURGEON Nicola «Scotland in EU », Édimbourg, Scottish Government, 25 juillet 2016.

40. www.whatscotlandthinks.org, consulté le 5 septembre 2016.

41. WILSON Gordon, « Long Term Strategy for Independence », Options for Scotland, juillet 2016.

42. Ce constat est partagé par Dennis Canavan, ex-travailliste et ex-dirigeant du conseil consultatif de Yes Scotland, GARDHAM Magnus, « Canavan Declares Time to Bring on New Poll », The Herald, 28 juin 2016, p. 1.

43. HC, Hansard, 6 septembre 2016, col. 84, Patrick Grady.

44. MURKENS Jo Eric, JONES Peter et KeATING Michael, Scottish Independence: a Practical Guide, Édimbourg, Edinburgh University Press, 2002, p. 130.

45. L'Écosse a même été comparée à la Grèce. MAHONAY Daniel et KNOX Tim, « Scotland : Could it Become Greece without the Sun?», Economic Bulletin, Londres, Centre for policy studies, $n^{\circ} 80,2$ juillet 2016.

46. Herald, « Mandarin : Brexit can be Scots' Moment », 9 juillet 2016, p. 6.

47. Après avoir posé de multiples questions relatives à la libre circulation pendant l'été, ils ont mis l'accent sur le marché unique, source de discorde parmi les Conservateurs anglais. Mais Sturgeon manque également de clarté, entre appartenance ou accès privilégié à ce dernier. SP, Official Report, 7 septembre 2016, col. 20 ; 14 septembre 2016, col. 20.

48. SP, EERC, Report, 12 septembre 2016, col. 10. 
49. À l'automne 2016, il est même apparu que même le «rapatriement » des compétences de Bruxelles à Londres dans les domaines gérés par le Parlement écossais (comme la pêche) ne se traduirait peut-être pas automatiquement par une dévolution à ce dernier; le gouvernement écossais devrait donc également solliciter ce transfert.

50. Ces incertitudes avaient été préjudiciables en 2014, GALlaGHER Jim, The Brexit Shambles: Charting a Path through the Rubble, Londres, Constitution unit, 21 juillet 2016.

\section{RÉSUMÉS}

Le 23 juin 2016, les Écossais ont choisi de rester dans l'UE, à la différence de la majorité des électeurs du Royaume-Uni. Cette divergence était prévisible car la plupart des hommes politiques écossais avaient mené campagne en faveur de l'UE, présentée comme une chance et non comme une menace. Le SNP, au pouvoir, a saisi cette occasion pour évoquer l'organisation d'un second référendum d'autodétermination. Les Écossais pourraient donc devoir choisir entre deux unions (UE et Royaume-Uni) dans un contexte très incertain.

On June $23^{\text {rd }}$ the Scots voted to remain in the UE contrary to the majority of voters in the UK. This discrepancy was to be expected as most senior politicians in Scotland had campaigned for the EU regarded as an asset and not as a threat. The ruling SNP has seized the opportunity to put a second referendum on Scotland's independence on the agenda. The Scots might thus have to choose between two unions -the EU and the UK- in spite of the prevailing uncertainty.

INDEX

Mots-clés : Écosse, référendum, UE, indépendance, dévolution, Nicola Sturgeon

Keywords : Scotland, referendum, EU, independence, devolution, Nicola Sturgeon

\section{AUTEUR}

\section{EDWIGE CAMP-PIETRAIN}

Université de Valenciennes 